\title{
DESENVOLVIMENTO DO TESTE DE PLANEJAMENTO TORRE DE LONDRES - VERSÃO BRASILEIRA (TOL-BR)
}

\section{DEVELOPMENT OF PLANNING TEST IN THE TOWER OF LONDON - BRAZILIAN VERSION (TOL-BR)}

\begin{abstract}
Resumo
Funções executivas permitem ao indivíduo resolver problemas complexos e lidar com novos contextos. Dentre elas, o planejamento é considerado uma função de alta ordem que está fortemente relacionada aos circuitos frontoestriatais, cujos déficits podem ser encontrados em diversos transtornos, como autismo, transtorno do déficit de atenção com hiperatividade (TDAH) e depressão. Um dos principais instrumentos para avaliar planejamento é a Torre de Londres (ToL), que, apesar de boas qualidades, tem apresentado problemas como efeito teto e baixa capacidade discriminativa em indivíduos normais. O presente estudo visa avaliar as propriedades psicométricas de uma versão computadorizada, brasileira, da Torre de Londres (ToL-BR). Foram testadas todas as possibilidades de itens possíveis no instrumento (35); após as exclusões dos itens com baixo poder discriminativo $\left(r_{p b} \ltimes 0,30\right)$, ficaram na ToL-BR um total de 19 itens. Os resultados preliminares, somados a estudos anteriores, sugerem que esse instrumento se mostra mais adequado para avaliar pessoas nos níveis inferior a médio das habilidades de planejamento.
\end{abstract}

Palavras-chave: Avaliação neuropsicológica, funções executivas, planejamento cognitivo.

\section{Abstract}

Executive functions allow individuals to solve complex problems and deal with new situations. Among these functions, planning is considered a high order function, strongly related to frontostriatal circuitry, and its deficits can be found in many disorders, such as autism, attention deficit hyperactivity disorder (ADHD) and depression. One of the instruments most widely used to assess such function is the Tower of London (ToL), which, despite good qualities, has been showing problems such as ceiling effect and low discriminative potential in normal individuals. The present study aims to assess the psychometric properties of a computerized Brazilian version of the Tower of London test (ToL-BR). All possible combinations (35) of items on ToL were tested; after exclusion of the less discriminative items, a total of 19 remained in ToL-BR. The preliminary results here presented, added to previous studies, suggest that this version of ToL is more adequate to assess individuals with inferior to medium planning skills.

Keywords: Neuropsychological assessment, executive functions, cognitive planning.

\section{INTRODUÇÃO}

Resolver problemas complexos e lidar com novos contextos depende de processos cognitivos de alta ordem, como, por exemplo, as funções executivas. Segundo Diamond ${ }^{1}$, podem ser consideradas funções executivas nucleares a memória operacional, o controle inibitório e a flexibilidade cognitiva, que subsidiam funções executivas complexas, como o planejamento, a resolução de problemas, o raciocínio dedutivo e a inteligência fluida. As funções executivas são consideradas a base do comportamento intencional e nos permitem a definição de objetivos, a antecipação e a seleção de etapas, a organização de recursos e o gerenciamento de tempo, monitorando o curso das ações e mudando o comportamento, se necessário. 


\section{ALEXANDRE LUIZ DE OLIVEIRA SERPA ${ }^{1}$, ALBERTO PENA PEREIRA TIMÓTEO², EMANUEL HENRIQUE GONÇALVES QUERINO², LEANDRO F. MALLOY-DINIZ ${ }^{3}$}

${ }^{1}$ Universidade Presbiteriana Mackenzie e Meta Cognitiv, São Paulo, SP. ${ }^{2}$ Universidade Federal de Minas Gerais (UFMG), Belo Horizonte, MG. ${ }^{3}$ Faculdade de Medicina, UFMG, Belo Horizonte, MG. Departamento de Saúde Mental, Universidade FUMEC, Belo Horizonte, MG.

A capacidade de planejar e resolver problemas está diretamente relacionada a uma série de domínios e contextos da vida quotidiana, como o desempenho acadêmico $^{2}$, o comportamento no trânsito $^{3}$ e a capacidade de gerir as próprias finanças ${ }^{4}$. Habilidades de planejamento menos eficazes também estão relacionadas à procrastinação e seus prejuízos ${ }^{5}$.

A capacidade de planejamento apresenta uma longa trajetória de desenvolvimento entre a infância até a idade adulta ${ }^{6}$. Além disso, do ponto de vista neurobiológico, depende da maturação e do bom funcionamento dos circuitos frontoestriatais? ${ }^{7}$. Comprometimentos nesses circuitos podem interferir na capacidade de definir objetivos, selecionar métodos, implementálos e monitorá-los, o que ocorre frequentemente em vários transtornos psiquiátricos. Déficits nas funções de planejamento ocorrem no transtorno do espectro do autismo ${ }^{8}$, no transtorno do déficit de atenção com hiperatividade (TDAH) $)^{9}$, no transtorno obsessivocompulsivo ${ }^{10}$, no transtorno bipolar ${ }^{11}$, na depressão ${ }^{12}$ e na esquizofrenia ${ }^{13}$. Portanto, as habilidades de planejamento são alvos essenciais para avaliação e intervenção em saúde mental.

Entre as medidas neuropsicológicas para avaliação do planejamento está a Torre de Londres (ToL) desenvolvida por Shallice ${ }^{14}$. A tarefa consiste na solução de problemas em que, com o menor número de movimentos possível, o voluntário deverá posicionar três esferas de cores diferentes em três pinos de tamanhos diferentes para que elas fiquem em uma configuração-alvo apresentada ao sujeito. A dificuldade da tarefa consiste em realizar a ordenação correta das esferas dentro de uma quantidade máxima de movimentos. Desde seu surgimento, a tarefa tem se mostrado bastante útil na identificação de problemas de planejamento em pessoas com comprometimentos envolvendo lesões préfrontais $^{14-16}$. Do mesmo modo, pacientes com transtornos neuropsiquiátricos também apresentam dificuldades nessa tarefa, como os indivíduos com TDAH ${ }^{17}$, demência frontotemporal e Alzheimer ${ }^{18}$, transtorno bipolar ${ }^{12}$, epilepsia ${ }^{19}$, entre outros.

Existem diversas versões da ToL, que variam em quantidade problemas empregados, formas de medidas utilizadas e pontuações. Usualmente, a ToL é composta por 12 problemas apresentados em ordem crescente de dificuldade em função do número de movimentos necessários para se alcançar a posição-alvo (de dois a cinco movimentos). Para cada problema, o sujeito tem três chances de solução, com a menor quantidade de movimentos possível; a pontuação pode variar entre 3 (se conseguir resolver o problema na primeira apresentação) e 0 pontos (se não conseguir resolver em nenhuma das apresentações). Existem adaptações para essa versão em vários países, como Itália ${ }^{20}$, República Checa $^{21}$ e Brasil $^{22-24}$. No entanto, alguns estudos reportam que a tarefa é bastante fácil e apresenta efeito teto se aplicada em indivíduos da população geral ${ }^{25}$.

No intuito de aumentar a eficiência do paradigma da ToL, diversos autores têm proposto modificações no sistema de administração e pontuação ${ }^{25}$, algumas das quais em versões computadorizadas ${ }^{26}$, possuindo variações em quantidade de problemas e medidas (por exemplo, velocidade de realização da tarefa). Com o objetivo de apresentar uma versão mais eficiente do paradigma da ToL, desenvolvemos uma versão computadorizada da tarefa (ToL-BR), composta por todas as configurações possíveis de posições das três bolas nos três pinos (um total de 36 problemas, com o primeiro deles usado apenas como exemplo). Nessa versão, os problemas podem ser resolvidos a partir de um movimento máximo (problemas mais fáceis) e sete movimentos máximos (problemas mais difíceis). No estudo inicial com a tarefa ${ }^{27}, 91$ adultos brasileiros foram submetidos a essa versão computadorizada e à versão tradicional de madeira com 12 problemas fundamentados na proposta de Krikorian ${ }^{28}$. Nesse estudo, a correlação entre os escores das duas versões foi moderada ( $r=0,42$, p < 0,05); no entanto, a ToL-BR apresentou uma consistência interna mais adequada do

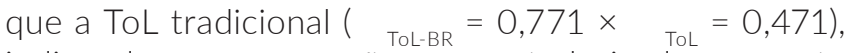
indicando que a versão computadorizada aparenta maior fidedignidade que a tradicional. Tal resultado foi congruente com a literatura, já que a versão tradicional de 12 itens tende a ter uma consistência interna menor que versões ampliadas ${ }^{29,30}$.

O objetivo deste estudo consiste em analisar as propriedades psicométricas dos diferentes itens da ToL$\mathrm{BR}$, selecionando os itens com melhores propriedades para a composição de uma tarefa capaz de avaliar de forma eficiente o planejamento em adultos brasileiros. 


\section{ARTIGO ORIGINAL}

ALEXANDRE LUIZ DE OLIVEIRA SERPA

ALBERTO PENA PEREIRA TIMÓTEO

EMANUEL HENRIQUE GONCCALVES QUERINO

LEANDRO F. MALLOY-DINIZ
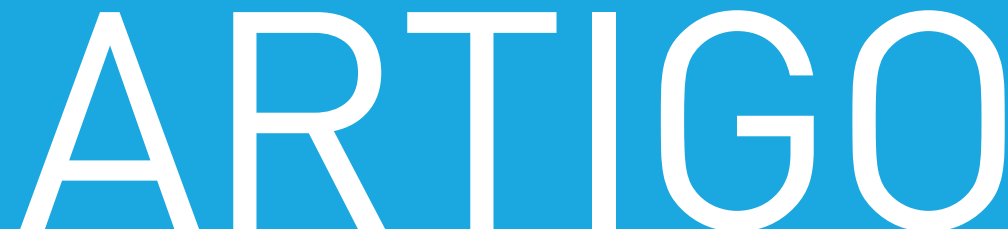

\section{MÉTODO}

\section{Participantes}

Participaram do estudo 179 indivíduos com idade entre 15 e 60 anos de idade (média $=34$ anos, desvio padrão $=12$ anos), sendo 51,9\% ( $n=93$ ) do sexo feminino $e$ $48,1 \%$ do sexo masculino ( $n=86$ ). Em relação ao nível de escolaridade, $57,5 \%$ estavam matriculados ou possuíam ensino superior, 9,5\% tinham completado o ensino médio e 8,4\% não informaram. Os demais possuíam alguma pós-graduação completa.

Instrumento: Torre de Londres, versão brasileira computadorizada (ToL-BR)27

Para a versão computadorizada da ToL, foi utilizado um software de uma plataforma virtual particular desenvolvidapelosautores(Figura1). Tal plataforma opera

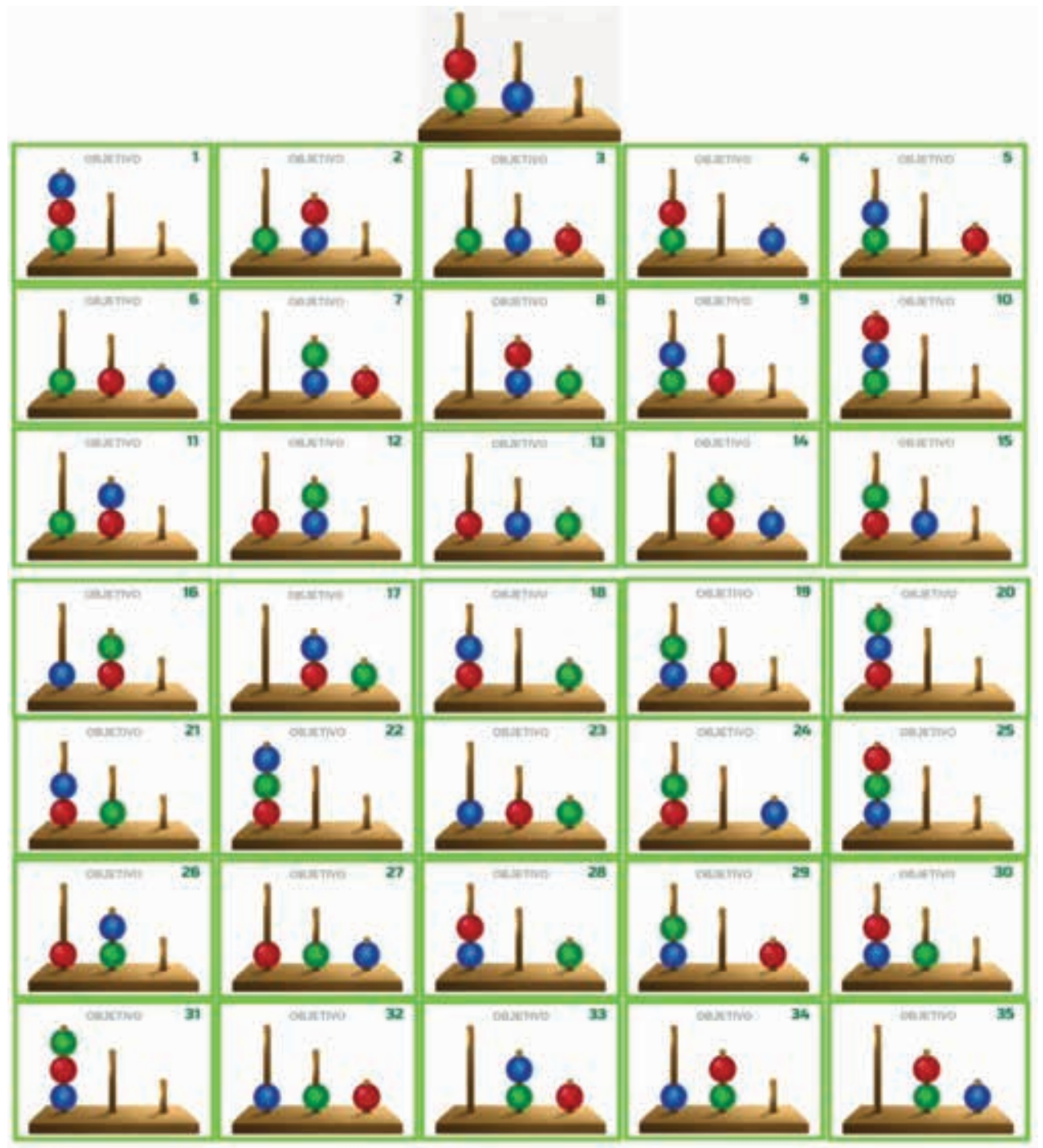

Figura 1 - Configuração inicial (no topo) e listagem das posições dos 35 problemas da Torre de Londres, versão brasileira computadorizada (ToL-BR). 


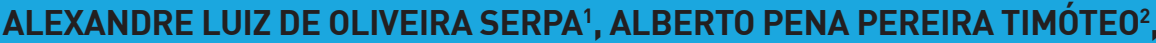 EMANUEL HENRIQUE GONÇALVES QUERINO², LEANDRO F. MALLOY-DINIZ ${ }^{3}$}

1 Universidade Presbiteriana Mackenzie e Meta Cognitiv, São Paulo, SP. ${ }^{2}$ Universidade Federal de Minas Gerais (UFMG), Belo Horizonte, MG. ${ }^{3}$ Faculdade de Medicina, UFMG, Belo Horizonte, MG. Departamento de Saúde Mental, Universidade FUMEC, Belo Horizonte, MG.

em navegadores com suporte a HTML5 e JavaScript, portanto uma conexão com a internet se faz necessária à aplicação. As regras de pontuação da tarefa foram as mesmas usadas por Krikorian et al. ${ }^{28}$, sendo 3 pontos para a resolução na primeira tentativa, 2 para a segunda, 1 para a terceira e 0 no caso de insucesso em todas as tentativas. A tarefa foi cautelosamente desenhada para que represente visualmente o instrumento original físico. A interação do usuário com a plataforma se dá por meio do mouse: com este, deve-se clicar na esfera desejada e, após isso, na haste em que se deseja posicionar a esfera, contando assim um movimento. Na ToL-BR, os sujeitos resolveram 35 problemas diferentes, que consistem em todas as permutações possíveis das três esferas nos três pinos. É importante ressaltar que, em razão disso, 12 dos problemas apresentados nessa versão são necessariamente idênticos aos realizados pelos sujeitos que executam a versão de Krikorian et al. ${ }^{28}$.

\section{Aspectos éticos}

Após consentirem voluntariamente a participação no estudo e concordarem com o termo de consentimento livre e esclarecido, os sujeitos foram submetidos à ToL-BR. O projeto de pesquisa foi aprovado pelo Comitê de Ética em Pesquisa da Universidade Federal de Minas Gerais (UFMG; processo CAAE: 53477016.6.0000.5149).

\section{Análises estatísticas}

Análises descritivas foram empregadas na versão de 35 itens para verificar a distribuição de respostas para cada uma das tarefas. Por se tratar de itens novos, foi verificada a presença ou a ausência de missing cases e padrões de respostas inesperados. A correlação pontobisserial foi utilizada para verificar a contribuição de cada item ao escore final de cada participante, de modo que itens com contribuição negativa ou baixa contribuição deveriam ser excluídos devido à baixa capacidade de discriminar os respondentes. Tais análises foram realizadas no software $\mathrm{R}^{31}$, com o pacote psych $^{32}$.

A versão resultante dessas análises preliminares foi avaliada em relação à sua dimensionalidade por meio do software FACTOR, versão 10.9.02 ${ }^{33}$. Para investigar quantos fatores seriam possíveis para o conjunto de dados, optou-se inicialmente por realizar uma análise paralela, utilizando a implementação otimizada de Timmerman \&
Lorenzo-Seva ${ }^{34}$. Considerando a literatura sobre a ToL, era esperado que o instrumento tivesse uma estrutura unidimensional. Assim, optou-se também por realizar a análise closeness to unidimensionality assessment ${ }^{35}$.

Em seguida, a estrutura fatorial sugerida pela análise paralela foi testada por meio de uma análise fatorial sem restrições, com o uso do estimador robust unweighted least squares (RULS) e correção de qui-quadrado robust mean and variance-scaled ${ }^{36}$. Além de verificar a carga fatorial de cada item no fator correspondente, foram utilizados também índices de ajuste do modelo, a saber, o comparative fit index (CFI 70,90$)$, o non-normed fit index (NNFI 70,90 ) e o root mean square error of approximation (RMSEA $\ltimes 0,05)$, utilizando os cortes propostos por Kline $^{37}$. Nessa etapa, a fidedignidade da escala seria verificada por meio do ômega de McDonald e do alfa de Cronbach.

Para testar a configuração final da escala, foi realizada ainda uma análise fatorial restrita, utilizando o software $\mathrm{R}^{31}$ por meio dos pacotes lavaan ${ }^{38}$ e semPlot ${ }^{39}$. A ordinalidade dos itens foi considerada e, por essa razão, utilizouse o weighted least square mean and variance adjusted (WLSMV), com correção de Satorra-Bentler para o quiquadrado. Assim como na análise fatorial não restrita, foram avaliadas a carga fatorial e os índices de ajuste do modelo. É importante frisar que há uma diferença de nomenclatura no software R, onde o NNFI é chamado de Tucker-Lewis index (TLI), sem que haja alteração no referencial de corte que será utilizado ${ }^{37}$.

A fim de consolidar a construção da medida da ToL-BR, optou-se por realizar uma análise dos itens, utilizando métodos da família da teoria de resposta ao item. Dois modelos politômicos foram ajustados ao conjunto de dados, um modelo de escalas graduais ${ }^{40}$ e um modelo de créditos parciais ${ }^{41}$. Os pacotes utilizados foram o eRm ${ }^{42}$, o RaschSampler ${ }^{43}$ e o mirt ${ }^{44}$. A qualidade do ajuste dos itens aos modelos foi testada por meio dos índices de ajuste das pessoas e itens (infit e outfit), pelo teste de Wald para a eliminação de itens e pelo teste de razão de verossimilhança de Andersen ${ }^{45}$. É também apresentada a fidedignidade para todos os pontos da escala e o índice de separação de pessoas.

\section{ResUltados}


ALEXANDRE LUIZ DE OLIVEIRA SERPA ALBERTO PENA PEREIRA TIMÓTEO EMANUEL HENRIQUE GONÇALVES QUERINO LEANDRO F. MALLOY-DINIZ
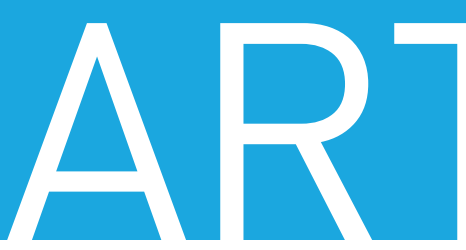

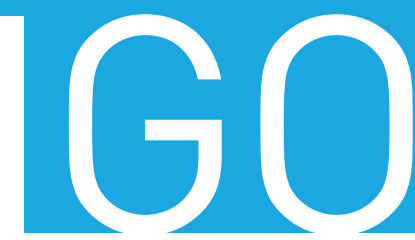

Considerando o universo de respostas possíveis para o instrumento, foi possível perceber, por meio das análises descritivas, que os itens 1, 2, 3, 4, 5, 6, 7, 8, 9, 10, 12, 14 e 15 foram respondidos corretamente, na primeira tentativa, por mais de $90 \%$ da amostra. Não foram encontrados valores significativos de missing data para nenhum item do instrumento. Por meio da correlação ponto-bisserial, observou-se que o item 10 tinha correlação negativa com o escore do teste; por essa razão, ele foi eliminado. Já os itens 1, 2, 3, 4, 5, 6, 7, 8, 9, 12, 14, 15, 18, 26 e 33 foram excluídos por apresentarem baixo poder discriminativo $\left(r_{p b} \ltimes 0,30\right)$, reduzindo o total de itens na escala para 19.

A análises descritivas dos itens mostraram que a média dos itens variou de 1,746 a 2,724, com variância entre 0,490 e 1,725. Os itens apresentaram uma tendência de assimetria positiva, dado que o valor mais alto possível (3 pontos) correspondia ao acerto na primeira tentativa de conclusão da tarefa. Considerando a distribuição e a ordinalidade dos itens, optou-se pela utilização da matriz de correlação policórica para o restante das análises.

A versão de 19 itens foi objeto de análise paralela, a qual sugeriu uma estrutura unidimensional para o conjunto de dados. Para o teste closeness to unidimensionality assessment, os resultados indicaram que dois índices tiveram valores que apontavam para unidimensionalidade: unidimensional congruence (UniCo) $=0,95 \pi 0,95$, e mean of item residual absolute loadings $(M I R E A L)=0,22 \ltimes 0,30$; e um índice não foi adequado: explained common variance $(E C V)=0,81 \pi 0,885$. Considerando as evidências encontradas e a literatura sobre a ToL, decidiu-se considerar como unidimensional o instrumento desenvolvido.

$\mathrm{Na}$ análise fatorial não restrita, considerando a existência de um fator, os índices de ajuste indicaram que o modelo unidimensional foi adequado para a versão de 19 itens, com CFI = 0,988, NNFI = 0,987 e RMSEA = 0,032 (0,010-0,050). Todos os itens tiveram saturação adequada no fator, e 31,5\% da variância dos dados foi explicada por eles. Em termos de fidedignidade, o ômega de McDonald foi igual a 0,875 e o alfa de Cronbach igual a 0,874, indicando que essa configuração possui evidência de fidedignidade adequada.

Este modelo foi então submetido a análise fatorial restrita robusta. Os índices de ajuste robusto do modelo se mostraram adequados [ $2=204,156$, grau de liberdade $(\mathrm{gl})=152$, fator de correção Satorra-Bentler $=0,710, \mathrm{CFI}=0,978, \mathrm{TLI}=0,976$, RMSEA $=0,037$ $(0,022-0,049)]$. Na Figura 2, estão disponibilizados os índices ajustados de saturação dos itens no fator latente para a ToL-BR.

A fim de consolidar a construção da medida da ToL-BR, optou-se por realizar uma análise dos itens, utilizando métodos da família da teoria de resposta ao item. Tanto o modelo de escalas graduais quanto o modelo de crédito parciais convergiram. Porém, a análise do ajuste dos itens, por meio dos testes de Wald para eliminação de itens e da razão de verossimilhança de Andersen, apontou que nem todos os itens apresentaram ajuste adequado para ambos os modelos, ainda que os índices de infit e outfit estivessem dentro dos parâmetros.

Uma análise detalhada das curvas características dos itens, com especial atenção para os thresholds das

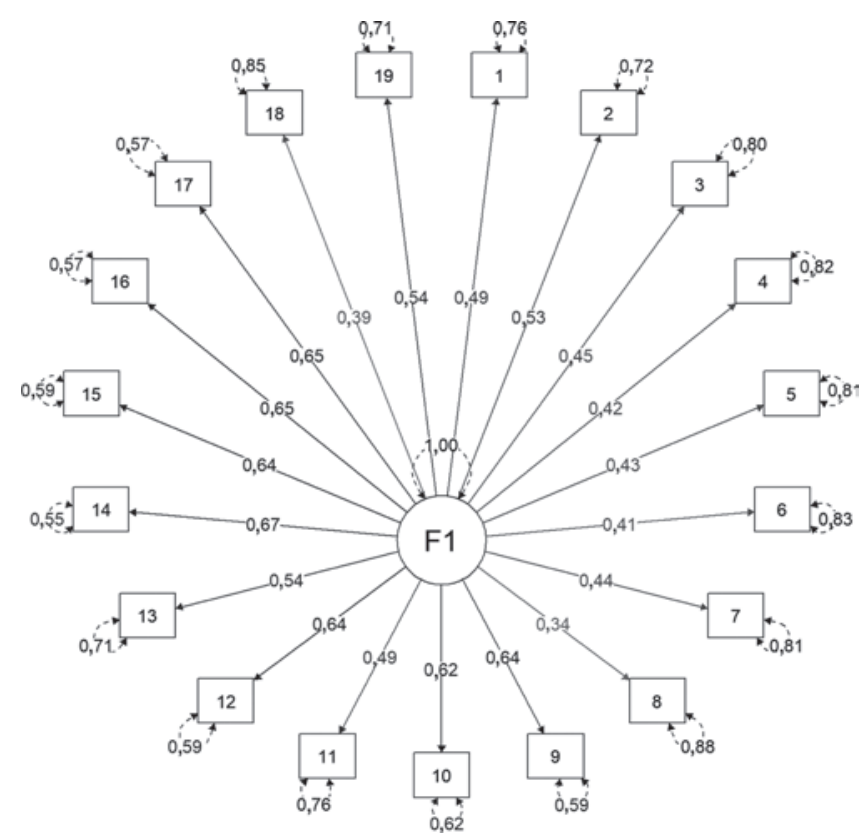

Figura 2 - Modelo unidimensional e cargas fatoriais da versão de 19 itens para o teste Torre de Londres, versão brasileira computadorizada (ToL-BR). F1 = fator 1 . Os números correspondem ao número do item na escala. 


\section{ALEXANDRE LUIZ DE OLIVEIRA SERPA', ALBERTO PENA PEREIRA TIMÓTEO², EMANUEL HENRIQUE GONÇALVES QUERINO², LEANDRO F. MALLOY-DINIZ ${ }^{3}$}

${ }^{1}$ Universidade Presbiteriana Mackenzie e Meta Cognitiv, São Paulo, SP. ${ }^{2}$ Universidade Federal de Minas Gerais (UFMG), Belo Horizonte, MG. ${ }^{3}$ Faculdade de Medicina, UFMG, Belo Horizonte, MG. Departamento de Saúde Mental, Universidade FUMEC, Belo Horizonte, MG.

pontuações extremas (3 e 0), indicou que a transição mais provável entre as pontuações é a da pontuação máxima para a mínima, de modo que é possível interpretar que as pontuações intermediárias poderiam não ser suficientemente informativas (Figura 3A). Com exceção dos itens 23, 24, 25 e 29, todos os itens apresentaram a transição dos itens da categoria 3 para a categoria 0.

No âmbito específico da tarefa da ToL-BR, isso significa dizer que a pontuação da prova como certo ou errado, sem necessidade de novas oportunidades para realização do exercício, ao menos com esse conjunto de itens, não implicaria prejuízo para a estimação da habilidade do indivíduo típico.

Para testar essa hipótese, foi delineado um modelo logístico simples de Rasch, considerando os acertos na primeira tentativa como 1 e acertos em outras tentativas ou erro como 0 . O modelo convergiu adequadamente, e os testes de Wald, Andersen e infit e outfit (Tabela
1) indicaram que os itens se ajustaram a esse modelo adequadamente.

Tabela 1 - Índices de infit e outfit dos itens para o modelo de Rasch

\begin{tabular}{cccccc}
\hline Item & $\mathbf{2}$ & $\mathbf{g l}$ & Valor de $\mathbf{p}$ & Outfit & Infit \\
\hline i11 & 158,854 & 180 & 0,870 & 0,878 & 0,965 \\
i13 & 153,724 & 181 & 0,930 & 0,845 & 0,933 \\
i16 & 156,567 & 181 & 0,905 & 0,860 & 0,961 \\
i17 & 219,323 & 180 & 0,024 & 1,212 & 1,143 \\
i19 & 175,965 & 180 & 0,571 & 0,972 & 1,025 \\
i20 & 200,875 & 181 & 0,148 & 1,104 & 1,059 \\
i21 & 197,584 & 181 & 0,189 & 1,086 & 1,082 \\
i22 & 231,475 & 180 & 0,006 & 1,279 & 1,076 \\
i23 & 182,726 & 181 & 0,450 & 1,004 & 1,006 \\
i24 & 162,359 & 181 & 0,837 & 0,892 & 0,924 \\
i25 & 168,515 & 181 & 0,738 & 0,926 & 0,991 \\
i27 & 175,177 & 181 & 0,608 & 0,963 & 0,953 \\
i28 & 181,991 & 181 & 0,465 & 1,000 & 1,022 \\
i29 & 147,772 & 181 & 0,966 & 0,812 & 0,884 \\
i30 & 164,009 & 180 & 0,798 & 0,906 & 0,950 \\
i31 & 170,183 & 179 & 0,669 & 0,945 & 0,882 \\
i32 & 158,036 & 181 & 0,890 & 0,868 & 0,924
\end{tabular}
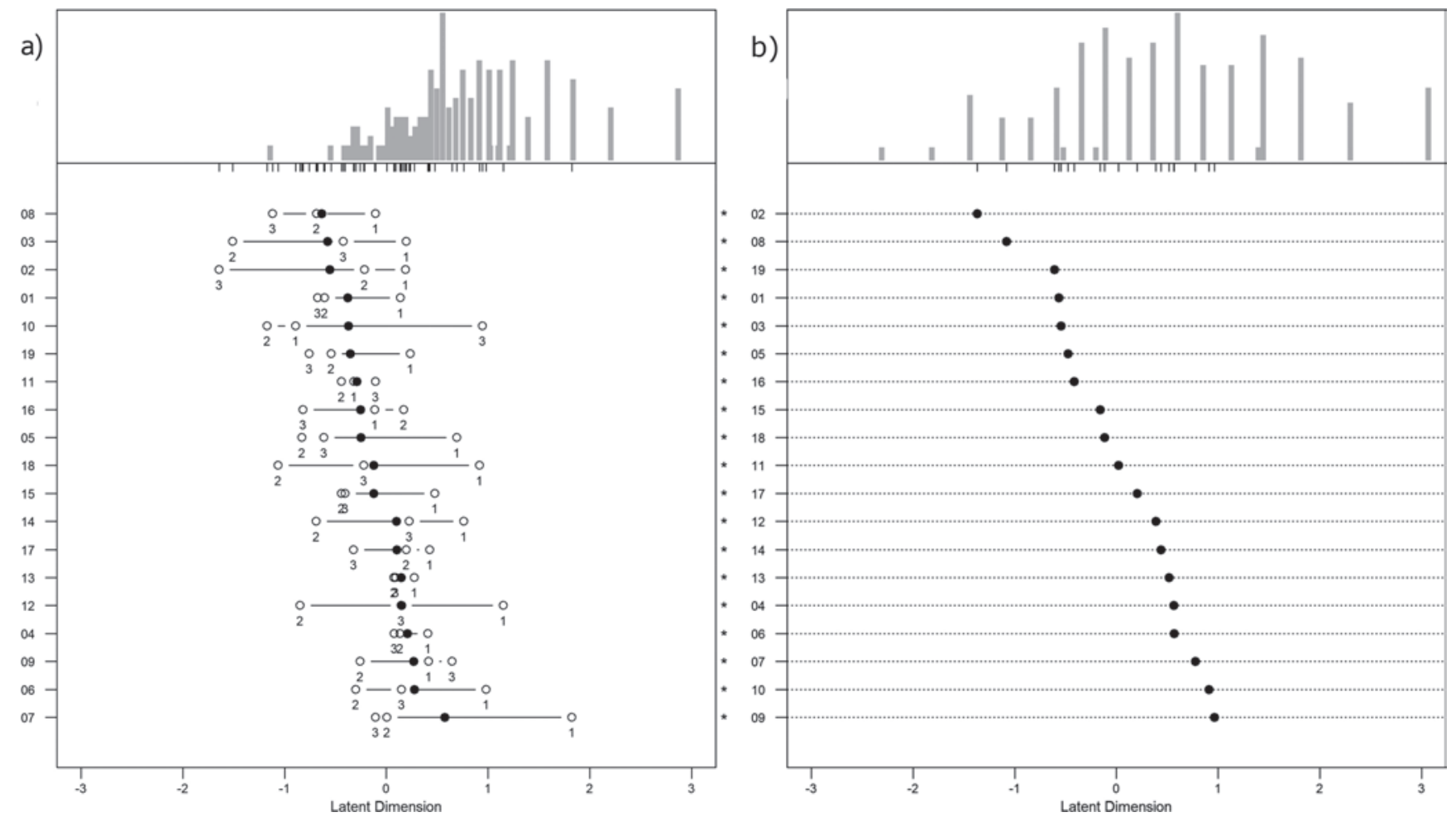

Figura 3 - Mapa de itens e pessoas para os itens da Torre de Londres, versão brasileira computadorizada (ToL-BR) nos modelos A) de créditos parciais e B) Rasch. 
ALEXANDRE LUIZ DE OLIVEIRA SERPA ALBERTO PENA PEREIRA TIMÓTEO EMANUEL HENRIQUE GONÇALVES QUERINO LEANDRO F. MALLOY-DINIZ

\begin{tabular}{llllll} 
i34 & 215,108 & 181 & 0,042 & 1,182 & 1,097 \\
i35 & 166,358 & 181 & 0,775 & 0,914 & 0,978 \\
\hline
\end{tabular}

$\mathrm{gl}=$ graus de liberdade.

O mapa de itens e pessoas (Figura 3B) apresenta a distribuição dos itens com base em sua dificuldade e a distribuição da amostra pelo continuum de habilidade. Observa-se que dois itens podem ser classificados como muito fáceis [itens 2 (antigo 13) e 6 (antigo 22)], e os demais itens apresentam um crescimento incremental no intervalo entre -1 e 1 desvio-padrão. Não foram observados itens que possam ser classificados como difíceis.

Em termos de fidedignidade, a escala apresentou um índice de separação de pessoas de 0,77 e superior a 0,60 para o intervalo entre aproximadamente -3 e 2 desvios padrão (Figura 4). O índice de separação de pessoas é uma medida similar ao alfa de Cronbach e pode ser interpretado da mesma maneira.

\section{DIscussão}

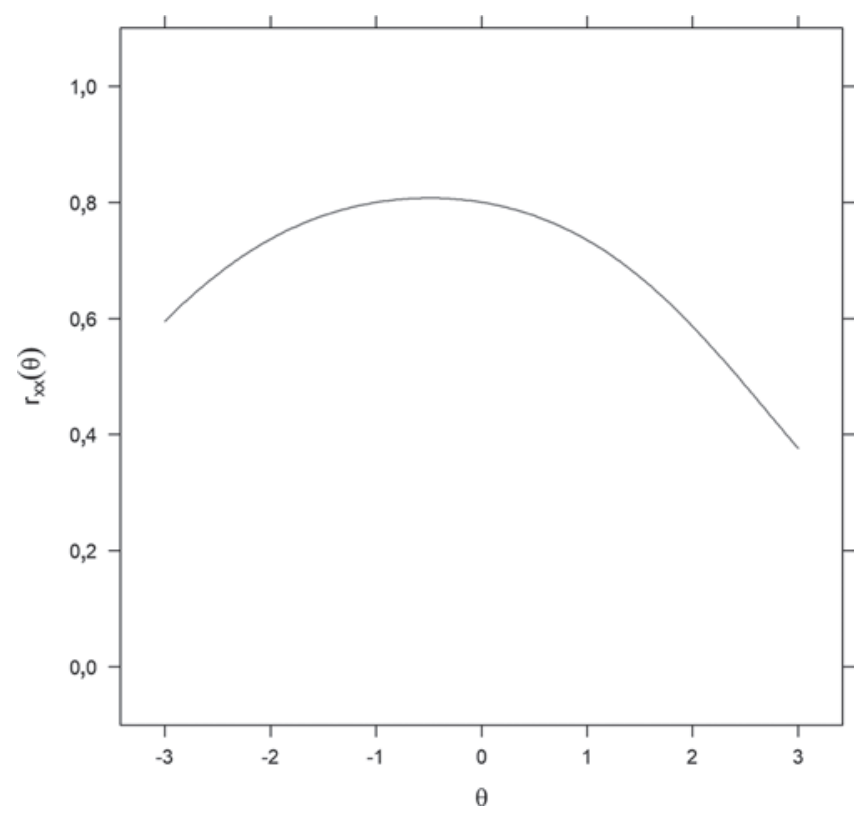

Figura 4 - Fidedignidade para todos os pontos da escala de habilidade da ToL-BR no modelo Rasch.
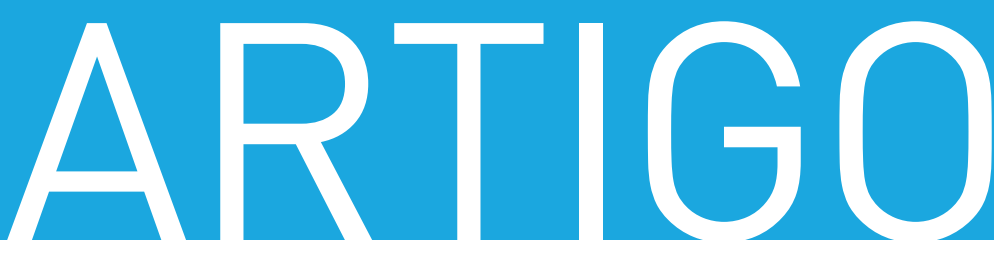

A ToL é uma tarefa clássica cujo objetivo é avaliar a habilidade de planejamento. Desde sua origem, existem diversas versões da tarefa que podem ser utilizadas tanto na neuropsicologia clínica quanto na pesquisa. Uma de suas versões mais populares se fundamenta no paradigma krikoriano ${ }^{28}$ e apresenta algumas limitações em relação a sua aplicabilidade, como, por exemplo, efeito teto ${ }^{25,46}$. O presente estudo teve como objetivo investigar as propriedades psicométricas de uma nova versão computadorizada da ToL (nomeada aqui como ToL-BR), que contou inicialmente com a criação de 35 itens em uma configuração de três hastes e três esferas. Desse conjunto, 19 itens apresentaram propriedades psicométricas adequadas e foram incluídos na versão final da tarefa.

Existem vários sistemas de correção para as diferentes versões da ToL. Michalec et al. ${ }^{21}$ apresentaram uma comparação entre quatro modos de pontuação para a tarefa, incluindo os dois modos originais propostos por Shallice ${ }^{14}$, o sistema de pontuação de Anderson et al. ${ }^{47}$ e o sistema de pontuação de Krikorian ${ }^{28}$. Esses sistemas usam o mesmo conjunto de 12 itens, mas diferem em função dos critérios e do uso do tempo total para a resolução de cada problema. De acordo com Michalec et al., todos os sistemas são úteis para a detecção de prejuízos no planejamento em amostras clínicas em pessoas com esquizofrenia e comprometimento cognitivo leve. Alguns autores, no entanto, propuseram novos sistemas de correção para avaliar déficits mais sutis e evitar o efeito teto. Portela et al. ${ }^{25}$, por exemplo, sugerem o uso de apenas uma tentativa para cada item, e que a pontuação total seja a soma dos movimentos corretos para concluir cada tentativa. Nesse estudo, os autores utilizaram os mesmos 12 itens da versão de Krikorian, e o sistema de correção proposto por eles apresentou melhores resultados do que o sistema clássico krikoriano, de três tentativas para cada item.

Com o mesmo objetivo de evitar o efeito teto presente na ToL, Raizner et al. ${ }^{46}$ desenvolveram uma versão ampliada da tarefa, com mais oito problemas, os quais exigiam de seis a oito movimentos para serem resolvidos. A inclusão desses itens tornou a tarefa mais difícil, dado que, na versão original, o item mais complexo requer cinco movimentos para ser resolvido 
com sucesso. Em comparação com a versão original, a ToL estendida se mostrou mais útil para discriminar diferenças no desempenho de crianças e adolescentes na tarefa.

Na mesma linha que os estudos anteriores, a nova versão da ToL proposta no presente estudo (ToLBR) apresenta sugestões de alterações tanto nos itens quanto no sistema de pontuação. Os resultados encontrados dão respaldo à hipótese de que uma escala de respostas de natureza dicotômica (certo ou errado) é tão informativa quanto a escala de respostas clássica da ToL, sendo também semelhante ao objetivo original relatado por Shallice ${ }^{14}$. Em complemento, os itens selecionados para a versão final da escala apresentam propriedades psicométricas adequadas, resultando em uma nova versão contendo 19 itens.

A ToL-BR apresentou evidências de validade quanto à estrutura interna alinhada com a literatura, replicando o modelo unidimensional recorrentemente encontrado (por exemplo, em Debelak et al. ${ }^{48}$ ). As evidências de fidedignidade apresentadas são iguais ou superiores às encontradas nas diferentes versões da ToL. Em um estudo de Humes et al. ${ }^{29}$ com a versão original da tarefa, o alfa de Cronbach encontrado foi igual a 0,25. Em contrapartida, Schnirman et al. ${ }^{30}$, trabalhando com uma versão revisada da tarefa composta por 30 itens, obtiveram um alfa igual a 0,79. Em um estudo anterior com a ToL-BR, Borges et al. ${ }^{27}$ compararam a versão informatizada de 35 itens com a versão de 12 itens de Krikorian, e a versão computadorizada apresentou um alfa de Cronbach de 0,71, enquanto a versão original obteve 0,47 no mesmo índice.

Pode-se concluir com essa análise preliminar que a tarefa se mostra mais adequada para avaliar pessoas nos níveis inferior a médio das habilidades de planejamento. Observe-se que a amostra do presente estudo permite a investigação das propriedades psicométricas preliminares da escala, sendo necessário que ela seja ampliada e diversificada e que os resultados sejam replicados, permitindo a extrapolação dos achados para a população brasileira. Além disso, a ausência de itens no estrato superior limita o papel do instrumento à função de rastreio. No futuro, pesquisas com populações clínicas podem trazer um entendimento mais aprofundado sobre os comportamentos dos itens nessa subpopulação. Evidências de validade relacionadas ao critério e à relação com outras variáveis também são necessárias para compreender as similaridades e dissimilaridades em relação à versão de lápis e papel.

Artigo submetido em 28/01/2020, aceito em 02/02/2020. Os autores informam não haver conflitos de interesse associados à publicação deste artigo.

Fontes de financiamento inexistentes.

Correspondência: Alexandre Serpa, Rua Piauí, 181, $10^{\circ}$ andar, Higienópolis, CEP 01241-001, São Paulo, SP. E-mail: alexandre@metacognitiv.com

\section{Referências}

1. Diamond A. Executive functions. Annu Rev Psychol. 2013;64:135-68.

2. Langberg JM, Dvorsky MR, Evans SW. What specific facets of executive function are associated with academic functioning in youth with attention-deficit/hyperactivity disorder? J Abnorm Child Psychol. 2013;41:1145-59.

3. Hayashi Y, Foreman AM, Friedel JE, Wirth O. Executive function and dangerous driving behaviors in young drivers. Transp Res Part F Traffic Psychol Behav. 2018;52:51-61.

4. Stro mba ck $C$, Lind $T$, Skagerlund $K$, Va stfja II D, Tingho g G. Does self-control predict financial behavior and financial well-being? J Behav Exp Finance. 2017;14:30-8.

5. Steel P, Svartdal F, Thundiyil T, Brothen $T$. Examining procrastination across multiple goal stages: a longitudinal study of temporal motivation theory. Front Psychol. 2018;9:327.

6. De Luca CR, Leventer RJ. Developmental trajectories of executive functions across the lifespan. In: Anderson V, Jacobs R, Anderson JP, eds. Neuropsychology, neurology, and cognition. Executive functions and the frontal lobes: a lifespan perspective. Milton Park: Taylor \& Francis; 2008. p. 23-56.

7. Van den Heuvel OA, Groenewegen HJ, Barkhof F, Lazeron RH, van Dyck R, Veltman DJ. Frontostriatal system in planning complexity: a parametric functional magnetic resonance version of tower of London task. Neuroimage. 2003;18:367-74. 
8. Olde Dubbelink LM, Geurts HM. Planning skills in autism spectrum disorder across the lifespan: a meta-analysis and meta-regression. J Autism Dev Disord. 2017;47:1148-65.

9. Boyer BE, Geurts HM, Van der Oord S. Planning skills of adolescents with ADHD. J Atten Disord. 2018;22:46-57.

10. Martoni RM, de Filippis R, Cammino S, Giuliani M, Risso G, Cavallini MC, et al. Planning functioning and impulsiveness in obsessive-compulsive disorder. Eur Arch Psychiatry Clin Neurosci. 2018;268:471-81.

11. Gvirts HZ, Braw $Y$, Harari H, Lozin M, Bloch $Y$, Fefer $K$, et al. Executive dysfunction in bipolar disorder and borderline personality disorder. Eur Psychiatry. 2015;30:959-64.

12. Fu L, Xiang D, Xiao J, Yao L, Wang Y, Xiao L, et al. Reduced prefrontal activation during the Tower of London and Verbal Fluency Task in patients with bipolar depression: a multi-channel NIRS study. Front Psychiatry. 2018;9:214.

13. Holt DV, Wolf J, Funke J, Weisbrod M, Kaiser S. Planning impairments in schizophrenia: specificity, task independence and functional relevance. Schizophr Res. 2013;149:174-9.

14. Shallice T. Specific impairments of planning. Philos Trans R Soc Lond B Biol Sci. 1982;298:199-209.

15. Owen AM, Downes JJ, Sahakian BJ, Polkey CE, Robbins TW. Planning and spatial working memory following frontal lobe lesions in man. Neuropsychologia. 1990;28:1021-34.

16. Nitschke K, Köstering L, Finkel L, Weiller C, Kaller CP. A Meta analysis on the neural basis of planning: activation likelihood estimation of functional brain imaging results in the tower of London task. Hum Brain Mapp. 2017;38:396-413.

17. Riccio CA, Wolfe ME, Romine C, Davis B, Sullivan JR. The tower of London and neuropsychological assessment of ADHD in adults. Arch Clin Neuropsychol. 2004;19:661-71.

18. Franceschi M, Caffarra P, Savarè R, Cerutti R, Grossi E, Tol Research Group. Tower of London test: a comparison between conventional statistic approach and modelling based on artificial neural network in differentiating fronto-temporal dementia from Alzheimer's disease. Behav Neurol. 2011;24:149-58.

19. Giovagnoli AR, Parente A, Didato G, Deleo F, Villani F. Expanding the spectrum of cognitive outcomes after temporal lobe epilepsy surgery: a prospective study of theory of mind. Epilepsia. 2016;57:920-30.

20. Boccia M, Marin D, D'Antuono G, Ciurli P, Incoccia C, Antonucci G, et al. The tower of London (ToL) in Italy: standardization of the ToL test in an Italian population. Neurol Sci. 2017;38:1263-70.

21. Michalec J, Bezdicek O, Nikolai T, Harsa P, Jech $R$, Silhan P, et al. A comparative study of tower of London scoring systems and normative data. Arch Clin Neuropsychol. 2017;32:328-38.

22. Malloy-Diniz LF, Cardoso-Martins C, Nassif EP, Levy AM, Leite WB, Fuentes, D. Planning abilities of children aged 4 years and 9 months to $81 / 2$ years: effects of age, fluid intelligence and school type on performance in the tower of London test. Dement Neuropsychol. 2008;2:26-30.

23. de Paula JJ, Neves F, Levy Â., Nassif E, MalloyDiniz LF. Assessing planning skills and executive functions in the elderly: preliminary normative data for the tower of London test. Arq Neuropsiquiatr. 2012;70:828-30.

24. Souza R, Ignácio FD, Cunha FC, Oliveira DL, Moll J. [Contributions to the neuropsychology of executive behavior: performance of normal individuals on the tower of London and Wisconsin tests]. Arq Neuropsiquiatr. 2001;59:526-31.

25. Portella MJ, Marcos-Bars T, Rami-Gonzalez L, Navarro-Odriozola V, Gastó-Ferrer C, Salamero M. [Tower of London: mental planning, validity and the ceiling effect]. Rev Neurol. 2003;37:2103.

26. Luciana M, Collins PF, Olson EA, Schissel AM. Tower of London performance in healthy adolescents: the development of planning skills and associations with self-reported inattention and impulsivity. Dev Neuropsychol. 2009;34:46175.

27. Borges A, Andrade CM, Timóteo AP, Schlottfeldt CG, Querino, EH, Godoy VP, et al. O teste da Torre de Londres: comparação entre a versão 
${ }^{1}$ Universidade Presbiteriana Mackenzie e Meta Cognitiv, São Paulo, SP. ${ }^{2}$ Universidade Federal de Minas Gerais (UFMG), Belo Horizonte, MG. ${ }^{3}$ Faculdade de Medicina, UFMG, Belo Horizonte, MG. Departamento de Saúde Mental, Universidade FUMEC, Belo Horizonte, MG.

tradicional e a aplicação computadorizada. Rev Debates Psiquiatr. 2018;8:30-36.

28. Krikorian R, Bartok J, Gay N. Tower of London procedure: a standard method and developmental data. Journal of Clinical and Experimental Neuropsychology. 1994;16:840-50.

29. Humes GE, Welsh C, Retzlaff PD. Development of the Tower of London-revised. Assessment. 1998;5:355-60.

30. Schnirman GM, Welsh MC, Retzlaff PD. Development of the tower of London-revised. Assessment. 1998;4:355-60.

31. R Core Team. R: a language and environment for statistical computing [Internet]. 2019. www.Rproject.org/

32. Revelle W. Psych: procedures for personality and psychological research [Internet]. 2019. CRAN.Rproject.org $/$ package=psych

33. Lorenzo-Seva U, Ferrando PJ. Program FACTOR, v. 10.9.02. Software program [Internet]. 2019 May 2. psico.fcep.urv.es/utilitats/factor/index.html

34. Timmerman ME, Lorenzo-Seva U. Dimensionality assessment of ordered polytomous items with parallel analysis. Psychol Methods. 2011;16:20920.

35. Ferrando PJ, Lorenzo-SevaU. Assessing the quality and appropriateness of factor solutions and factor score estimates in exploratory item factor analysis. Educ Psychol Meas. 2018;78:76280.

36. Asparouhov T, Muthen B. Simple second order chi-square correction [Internet]. 2010 May 2. www.statmodel.com/download/WLSMV_new_ chi21.pdf
37. Kline R. Principles and practice of structural equation modeling. 4th ed. New York: The Guilford; 2015.

38. Rosseel Y. lavaan: an R package for structural equation modeling [Internet]. 2012 May 24 [cited 2010 Feb 26]. www.jstatsoft.org/v48/i02/

39. Epskamp S. semPlot: path diagrams and visual analysis of various SEM packages' output [Internet]. 2019 [cited 2020 Feb 26]. cran.rproject.org/web/packages/semPlot/index.html

40. Andrich D. A rating formulation for ordered responsecategories. Psychometrika. 1978;43:56173.

41. Masters GN. A Rasch model for partial credit scoring. Psychometrika. 1982;47:149-74.

42. Mair P, Hatzinger R, Maier MJ. eRm: Extended Rasch Modeling 1.0-0 [Internet]. 2019. erm.rforge.r-project.org/

43. Mair P, Hatzinger R, Verhelst ND. RaschSampler: rasch sampler. $\mathrm{R}$ package version 0.8$8 \quad$ [Internet]. 2015. CRAN.R-project.org/ package= RaschSampler

44. Chalmers RP. mirt: a multidimensional item response theory package for the R environment. J Stat Softw. 2012;48:1-29.

45. Golino HF, Gomes CMA, Amantes A, Coelho G. Psicometria contemporânea: compreendendo os modelos Rasch. Belo Horizonte: Livraria do Psicólogo; 2019.

46. Raizner RD. Raising the ceiling: the tower of London-extended version. Dev Neuropsychol. 2002;21:1-14.

47. Anderson P, Anderson V, Lajoie G. The Tower of London test: validation and standardization for pediatric populations. Clin Neuropsychol. 1996;10:54-65.

48. Debelak R, Egle J, Köstering L, Kaller CP. Assessment of planning ability: psychometric analyses on the unidimensionality and construct validity of the Tower of London (TOL-F). Neuropsychology. 2016;30:346-60. 